\title{
An Apple II package for computer-assisted telephone interviewing
}

\author{
STEVEN F. PHILIPP and CHARLES F. CICCIARELLA \\ University of North Carolina, Greensboro, North Carolina
}

\begin{abstract}
This paper describes an Apple II (an Apple-compatible) microcomputer package for a computer-assisted telephone interviewing (CATI) system. This system generates random telephone numbers within any number of exchanges that the user supplies, automatically dials the telephone, prompts the interviewer with each question, and records and permanently seaves each response. It also provides a printed copy of the collected data by frequency of response in each question category. The Apple II CATI package is readily available, inexpensive, and easy to program in the BASIC language.
\end{abstract}

Computer-assisted telephone interviewing (CATI) systems have been developed in recent years (Shure \& Meeker, 1974, 1978) to merge technological advances with the many advantages of telephone interviewing. Although these CATI systems have allowed a few major funded projects with priority access to large university computer systems to be accomplished, potential research projects without such monetary or physical resources have not considered CATI a practical research alternative. System start-up costs may also contribute to the apparent scarcity of CATI-survey research projects. Indeed, Shanks, Nicholls, and Freeman (1981) concluded that "for the foreseeable future, computer assisted telephone interviewing will involve special costs" (p. 137). They further observed that "approximately eight months of highly intensive work were required by three senior staff members and several assistants to design, program, and debug the programs employed" (p. 137). Clearly, alternatives to present CATI systems are required that are widely available, cost efficient, and easily programmed.

\section{PROGRAM FOR THE APPLE COMPUTER}

The Apple II CATI system described here is one solution to the apparent applications problems of current CATI operations. The Apple II is widely available (an estimated 700,000 Apple IIs are currently in use), it is relatively inexpensive (about $\$ 2,000$ for the system described here), and it is easily programmed in the BASIC language. The authors have prepared a readyto-use program, supplied on a disk, to handle many of the operational and analytical functions of a telephonesurvey research project.

The Apple II CATI package was designed to assist a

The Apple II CATI package is available for $\$ 35$. For specific information, please contact the senior author: Steven $F$. Philipp, School of Health, Physical Education, Recreation, and Dance, University of North Carolina, Greensboro, North Carolina 27412. broad range of researchers of differing backgrounds to conduct telephone surveys. The program guides the researcher through the steps of entering a series of questions and answers into disk files that allow survey data to be entered into the computer as the data are collected. The program randomly generates and dials telephone numbers within any combination of telephone exchanges selected by the user. It then displays questions and answers on the television monitor screen to be read to the interviewee, records responses, and prints a table showing the frequency of responses to each question at any time requested. The data files created by the program can also be used by other programs for more sophisticated statistical analysis or can even be transmitted to other computers for analysis. The balance of this report describes the technical requirements and procedures of the system in greater detail.

\section{Hardware Requirements}

The hardware consists of an Apple II Plus personal computer with at least $32 \mathrm{~K}$ of RAM, television monitor, floppy-disk drive, printer, direct-connect modem, telephone, and dual modular telephone jack. The printer used in developing and testing the program was the Integral Data Systems Paper Tiger Model 460G, and the modem was a D. C. Hayes Micromodem II. An Apple Ile or any Apple-compatible computer may be substituted for the Apple II Plus. The computer system must be assembled in its standard configuration, with the disk drive interfaced through peripheral slot number 6, the modem through slot 3 , and the printer through slot 1 . The modem must connect to a private telephone line through one port of the dual modular jack, and the telephone must be connected in parallel through the other port. The telephone company must be notified of the connection for the researcher to be in compliance with Federal Communications Commission regulations.

Program Development Procedures

Program subroutines were written in floating-point 
BASIC for entry and editing of interviews, storage and retrieval of interviews to and from disk memory, entry of telephone exchanges to be sampled, conduct of interviews, and analysis of survey data, and then were integrated into a single program controlled by a core or menu program. All logical and syntax errors that could be detected were then corrected, and a sample set of interview data was entered to verify that the program was mathematically accurate.

\section{Operational Procedures}

As soon as power has been applied to the computer with the program disk in the disk drive, the program is loaded and run and a menu appears on the monitor screen and lists options for writing an interview, recalling an interview from disk storage, entering a list of telephone exchanges, conducting an interview, resuming an interview, printing output, and terminating the program. All subroutines are selected from this menu and are returned to it when completed.

Write interview. When the "WRITE INTERVIEW" subroutine is selected, the user is given prompts that guide the entry of a complete interview consisting of a brief greeting, a series of questions and possible choices, and a closing statement. Although no question or other element of the interview may exceed 255 characters and no commas may be used, any number of questions (up to the storage capacity of the computer's RAM memory) and any number of possible choices per question may be entered. With $48 \mathrm{~K}$ of RAM available, the length of the interview may be in excess of 20,000 characters.

Recall interview. This subroutine is used to recall an interview that has been entered previously and stored on a diskette.

Enter exchange list. The TELEPHONE SURVEY program is designed to randomly generate telephone numbers within a list of telephone exchanges entered using this subroutine. This subroutine can also be used to cause the survey sample to be distributed among certain exchanges in any desired pattern by the entering of certain exchanges more than once. For example, if the user desires to obtain $60 \%$ of the sample from exchange " $379, " 30 \%$ from exchange " 275 ," and $10 \%$ from exchange " 855 ," a list of 10 exchanges in which " 379 " is repeated six times, " 275 " occurs three times, and " 855 " appears once may be entered. When telephone numbers are generated, the algorithm used causes the computer to randomly select one of the exchanges and then randomly generate a four-digit telephone number. The random-number-generation method used by the Apple computer is such that the number generated is truly unpredictable, even when used in a program loop, and, although the argument may be made that the number is not completely random in a mathematical sense, any error that may occur is certainly random with respect to the demographics of any telephone survey.
Table 1

Response Board

\begin{tabular}{lll}
\hline Busy Signal & $=$ & 1 \\
Rang, No Answer & $=$ & 2 \\
Call Back Later & $=$ & 3 \\
Interview Accepted & $=$ & 4 \\
Interview Declined & $=$ & 5 \\
\hline Enter Code ? & \\
\hline
\end{tabular}

Conduct interview. After an interview has been entered, this subroutine handles the actual conduct of the survey. The program operator should be ready to speak on the telephone to the interview subject when this subroutine is run. To initiate an interview, the computer generates and dials a telephone number, and displays a response board on the screen. Table 1 simulates the appearance of the response board.

The computer records the results of the telephone call at this point if an interview is not obtained. If an interview is accepted, the computer displays, in succession, each question and possible answer choices on the screen, and records the responses obtained. After the last question has been answered, a closing statement, which should be read to the subject before the call is terminated, is displayed. When the call is terminated, the operator may optionally end the interviewing session or initiate another call.

Resume interview. This subroutine enables the operator to resume collecting data, without losing any of the previous data, after the computer has been turned off.

Print output. This subroutine is used to print raw or analyzed data on paper. When it is activated, a second menu showing various output options appears on the screen.

The option "PRINT RAW DATA" is used to print the data in raw form, just as it is stored on the diskette. This is useful with open-ended surveys or in searches for trends among unsuccessful calls. The option "FREQUENCIES (CATEGORICAL ONLY)" prints the raw data as in the previous subroutine but also produces a table showing the frequency of each response to each question. It can be used only with categorical response surveys. The remaining two options provide access to analyses that may be programmed by the user.

\section{LIMITATIONS}

All the usually cited telephone-survey limitations (e.g., Blankenship, 1977; Groves, 1979) are associated with the Apple II package described here. There are, however, four notable limitations of special interest to the present program. First, the program is designed specifically for fixed-format questions, since categorical information is generally a more efficient means of collecting, coding, and processing information. Second, the Apple II program relies on the geographic integrity of 
the telephone prefix areas; however, such prefix areas are rarely tight and neat, and the researcher should be aware of the "irregularities" of a chosen telephone prefix. Third, the interviewers, at least at present, must be trained to overcome any acquired resistance to computer operations. Finally, the functioning of each microcomputer as a separate operational unit makes it complicated, although not impossible, to link several units together for large surveys in which simultaneous operations of several units are required.

\section{SUMMARY}

This paper has described an Apple II computerassisted telephone interviewing (CATI) system for telephone-survey research. It describes the equipment needed, the choices inherent in the system, and the details of the developed program. The system described should allow considerable expansion of CATI use by providing a low-cost, readily available, and easy-toprogram alternative to current systems. A survey researcher's primary concerns should be with hypothesis construction, methodological issues, and data analysis; he should not be burdened with computer-package design and programming considerations. The present Apple II package offers the researcher the advantages of computer-assisted telephone interviewing without many of the current resource consuming problems.

\section{REFERENCES}

Blankenship, A. B. Professional telephone surveys. New York: McGraw-Hill, 1977.

Groves, R. M. Surveys by telephone: A national comparison with personal interviews. New York: Academic Press, 1979.

Shanks, J. M., Nicholls, W. L., II, \& Freeman, H. E. The California disability survey: Design and execution of a computerassisted telephone study. Sociological Methods and Research, $1981,10,123-140$.

Shure, G. H., \& Meeker, R. J. Laboratory experimentation at the Center for Computer-Based Behavioral Studies. Behavior Research Methods \& Instrumentation, 1974, 6, 241-248.

Shure, G. H., \& Meeker, R. J. A minicomputer system for multiperson computer-assisted telephone interviewing. Behavior Research Methods \& Instrumentation, 1978, 10, 196-202.

(Manuscript received December 7, 1982; revision accepted for publication August 4, 1983.) 